\title{
Segregation of Ipsilateral Retinal Ganglion Cell Axons at the Optic Chiasm Requires the Shh Receptor Boc
}

\author{
Pierre J. Fabre, ${ }^{1,2}$ Tomomi Shimogori, ${ }^{3}$ and Frédéric Charron ${ }^{1,2,4}$ \\ ${ }^{1}$ Molecular Biology of Neural Development, Institut de Recherches Cliniques de Montréal, Montreal, Quebec, Canada H2W 1R7, ${ }^{2}$ Department of Medicine, \\ University of Montreal, Montreal, Quebec H3C 3J7, Canada, ${ }^{3}$ RIKEN Brain Science Institute, Wako-Shi, Saitama 351-0198, Japan, and ${ }^{4}$ Department of \\ Anatomy and Cell Biology, Department of Biology, Division of Experimental Medicine and Program in Neuroengineering, McGill University, Montreal, \\ Quebec H3A 2T5, Canada
}

The pattern of contralaterally and ipsilaterally projecting retinal ganglion cell (RGC) axons at the optic chiasm is essential for the establishment of binocular vision. Contralateral axons cross the chiasm midline as they progress from the optic nerve to the optic tract. In contrast, ipsilateral axons deviate from the chiasm and continue in the ipsilateral optic tract, avoiding the chiasm midline. The molecular mechanism underlying this phenomenon is not completely understood. Here we show that the Sonic Hedgehog (Shh) receptor Boc is enriched in ipsilateral RGCs of the developing retina. Together with the presence of Shh at the midline, this complementary expression pattern led us to hypothesize that Shh might repel ipsilateral RGC axons at the chiasm. Consistent with this hypothesis, we found that only Boc-positive RGC axons retract in vitro in response to Shh and that this response is lost in Boc mutant RGCs. In vivo, we show that Boc is required for the normal segregation of ipsilateral axons at the optic chiasm and, conversely, that Boc expression in contralateral RGCs prevents their axons from crossing the optic chiasm. Together, these results suggest that Shh repels ipsilateral RGC axons at the optic chiasm via its receptor Boc. This work identifies a novel molecular pathway required for the segregation of axons at the optic chiasm.

\section{Introduction}

At the optic chiasm, the majority of retinal ganglion cell (RGC) axons cross the midline and continue their progression toward the brain in the contralateral optic tract. However, in animal species with binocular vision, a subpopulation of RGC axons do not cross the midline and, instead, avoid the chiasm to follow the ipsilateral optic tract (Erskine and Herrera, 2007; Petros et al., 2008). RGCs which project ipsilaterally are located in the ventrotemporal (VT) part of the retina and the guidance of their axons at the optic chiasm is regulated by pathfinding cues expressed by the midline radial glial cells and by cells of the ventral diencephalon surrounding the chiasm. A crucial midline cue is Ephrin-B2, which repels ipsilaterally projecting axons expressing the Ephrin receptor EphB1 (Nakagawa et al., 2000; Williams et al., 2003). The combined inactivation of all gradients of EphB receptors in the retina of the EphB1;EphB2;EphB3 triple mutant indicates

\footnotetext{
Received July 30, 2009; revised 0ct. 29, 2009; accepted Nov. 5, 2009.

This work was supported by grants from the Canadian Institutes of Health Research, the Peter Lougheed Medical Research Foundation, the Fonds de Recherche en Santé du Québec, and the Canada Foundation for Innovation. We are grateful to S. Brown, M. Cayouette, C. Jolicoeur, A. Kania, M. Lévesque, S. K. McConnell, S. Morin, A. Okada, E. Ruthazer, J. Sandink, and D. Van Meyel for providing advice and/or reagents. We thank J. Barthe, J. Cardin, A. Daigneault, and S. Morin for expert technical assistance and J. Pham for schematics. The $4 \mathrm{D} 7$ and $2 \mathrm{H} 3$ antibodies were obtained from the Developmental Studies Hybridoma Bank developed under the auspices of the National Institute of Child Health and Human Development and maintained by the University of lowa. We thank M. Cayouette, A. Kania, and P. T. Yam for critical comments on this manuscript.

Correspondence should be addressed to Dr. Frédéric Charron, Molecular Biology of Neural Development, Institut de Recherches Cliniques de Montréal, 110 Pine Avenue West, Montreal, QC H2W 1R7, Canada. E-mail: Frederic. Charron@ircm.qc.ca.

DOI:10.1523/JNEUROSCI.3778-09.2010

Copyright $\odot 2010$ the authors $\quad 0270-6474 / 10 / 300266-10 \$ 15.00 / 0$
}

that EphB receptors account for the formation of $\sim 50 \%$ of the ipsilateral projections in the mouse (Williams et al., 2003). Importantly, the residual $50 \%$ of the ipsilateral projections in these mutants still correctly originates from the VT retina, suggesting that other factors are still present to guide these ipsilateral RGC axons. These and other experiments (García-Frigola et al., 2008) have led to the hypothesis that ipsilateral RGC axons from the VT retina are also guided by EphB-independent mechanisms at the chiasm.

The secreted protein Shh is a morphogen which can also function as an axon guidance molecule (Charron et al., 2003; Charron and Tessier-Lavigne, 2005). Shh is expressed in the diencephalic ventral midline (Torres et al., 1996; Marcus et al., 1999; Wallace and Raff, 1999; Trousse et al., 2001) and it has been suggested to act as a repellent to guide RGC axons through the optic chiasm (Trousse et al., 2001). More recently, in vivo experiments that inhibit the Shh signaling mediator Smoothened (Smo) in the developing mouse visual system suggested that Shh controls the pathfinding of contralaterally projecting RGC axons in a cellautonomous way (Sánchez-Camacho and Bovolenta, 2008). However, contralaterally and ipsilaterally projecting RGCs both express components of the Shh signaling pathway, and these experiments did not assess whether ipsilaterally projecting RGC axons are normally guided at the chiasm under these conditions. Therefore, whether Shh guides ipsilateral projections remains unknown. Furthermore, the identity of the Shh receptor involved in the guidance of RGC axons has not been determined.

To address these two questions, we have taken advantage of our previous finding that Boc is a Shh receptor required for the 


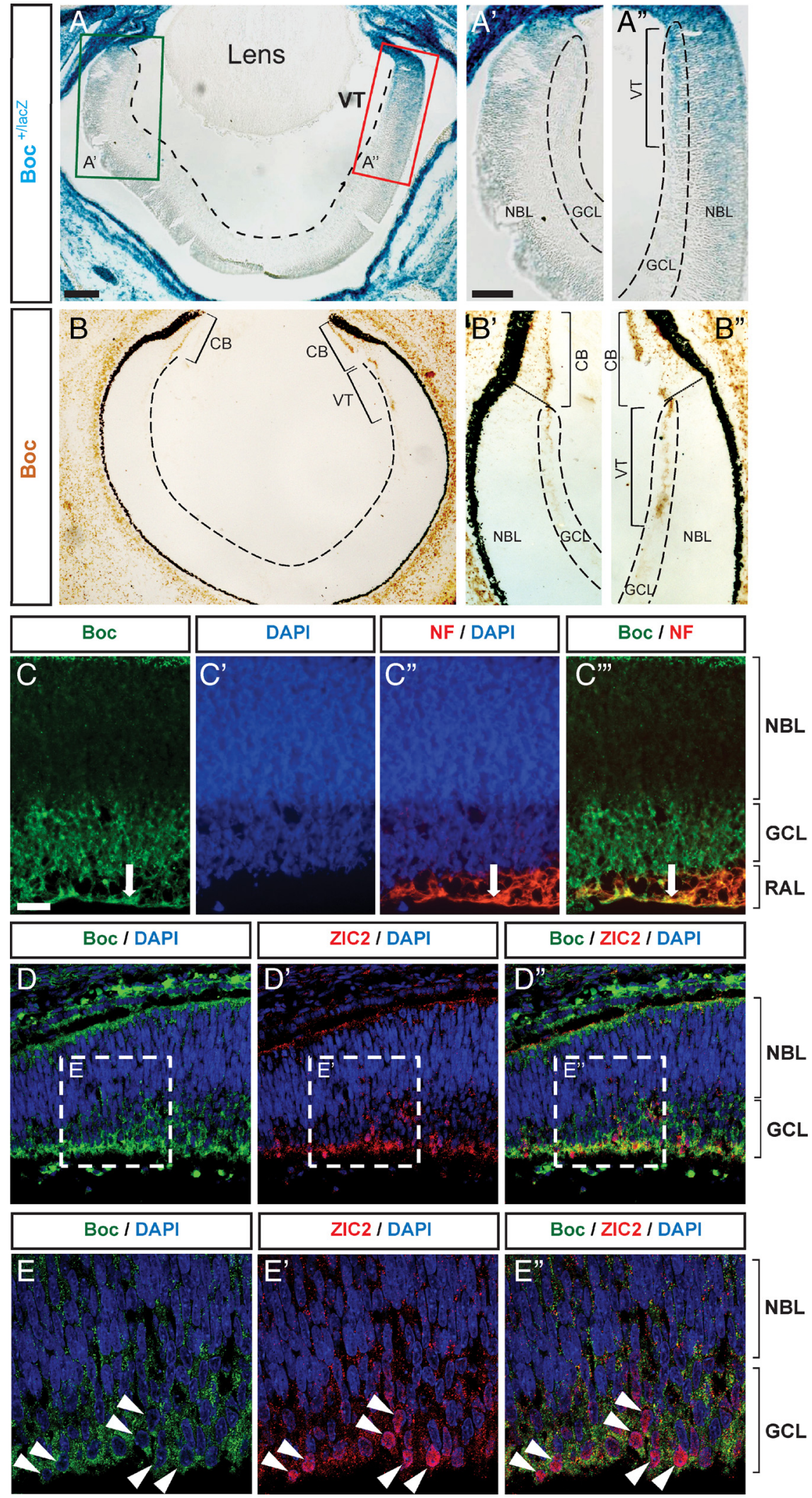

Figure 1. Boc is preferentially expressed in ipsilateral RGCs. $A-B^{\prime \prime}$, Boc expression detected by $\beta$-gal histochemistry in $B o c^{+}{ }^{\text {lac }}$ mice $\left(A-A^{\prime \prime}\right)$ and immunohistochemistry with an antibody against Boc (goat anti-Boc) in E15 horizontal section of the mouse retina ( $\left.\boldsymbol{B}-\boldsymbol{B}^{\prime}\right)$. Both methods show a strong enrichment of Boc in theVT retina as shown in $A^{\prime \prime}$ and $\boldsymbol{B}^{\prime \prime}$. C $\left(\boldsymbol{C}^{\prime \prime}\right.$, Boc (green, rabbit anti-Bocantibody) is expressed by RGC cell bodies in the GCL and is present in the RAL of theVT. Boccolocalizes with neurofilament (NF) on the axons of RGCs in the RAL (arrow; $C-C^{\prime \prime \prime}$ ) (horizontal sections of E17 rat retina). D- $\boldsymbol{D}^{\prime \prime}$, Zic2-positive cells (red) colocalize with Boc (green, goat anti-Boc antibody) in the VT crescent of E15 mouse retina (horizontal sections). Higher magnification of the boxed regions from $\boldsymbol{D}$ and $\boldsymbol{D}^{\prime}$ shows thenuclear localization of Zic 2 surrounded by membranous expression of Bocin the GCL (E-E').CB, Ciliary body. Scale bars: $A, 100 \mu \mathrm{m} ; A^{\prime}, A^{\prime \prime}, 50 \mu \mathrm{m} ; C^{\prime}-C^{\prime \prime}, 20 \mu \mathrm{m}$. guidance of commissural axons by Shh (Okada et al., 2006). We show that Boc is enriched in ipsilateral RGCs of the developing retina and that, in vitro, Boc is required for RGC axons to respond to Shh. In vivo, Boc is required for the normal segregation of ipsilateral axons at the optic chiasm and its expression in contralateral RGCs prevents their axons from crossing the optic chiasm. Together, these results suggest that Shh repels ipsilateral RGC axons at the optic chiasm via its receptor Boc. This work identifies a novel molecular pathway required for the segregation of axons at the optic chiasm.

\section{Materials and Methods}

Animals. Timed pregnant Sprague Dawley rats were obtained from Charles River Canada. C57BL/6J mice were maintained in a specificpathogen-free animal facility (Institut de Recherches Cliniques de Montréal, Montreal, Quebec, Canada). Boc $c^{+ \text {llacZ }}$ and Cdon ${ }^{+ \text {/lac } Z}$ mice were kindly provided by A. Okada and S. K. McConnell (Stanford University, Palo Alto, CA) (Okada et al., 2006) and were maintained on a C57BL/6J background. Embryonic day 0 (E0) was defined as midnight of the night before a plug was found. All animal work was performed in accordance with the Canadian Council on Animal Care Guidelines.

Reagents. Recombinant purified human ShhN (C24II; R\&D Systems) was used at $10 \mathrm{~nm}$, a concentration which induces efficient RGC axon retraction (Fig. S4, available at www.jneurosci.org as supplemental material). Smoothened antagonist (SANT-1) was purchased from Calbiochem and used at a final concentration of $137 \mathrm{~nm}$ (Chen et al., 2002).

Retinal explant cultures. Eight-chamber culture slides (BD Falcon) were coated with 10 $\mu \mathrm{g} / \mathrm{ml}$ poly-L-lysine (Sigma) for $2 \mathrm{~h}$ at room temperature, washed three times with water, and then coated overnight at $37^{\circ} \mathrm{C}$ with 6 $\mu \mathrm{g} / \mathrm{ml}$ laminin (Invitrogen) in PBS. Retinas were dissected from E17.5-E18.5 rat embryos, or from E15.5-E17.5 mouse embryos. Each retina was cut into $\sim 50$ explants (average size of $300 \mu \mathrm{m}$ ). Explants were then transferred to the culture slides and cultured in serumfree medium (Neurobasal and DMEM/F12), supplemented with B27 (Invitrogen) and N2 (Invitrogen). Retinal explants were then maintained at $37^{\circ} \mathrm{C}, 7.5 \% \mathrm{CO}_{2}$. Eighteen to twentytwo hours after being plated, $10 \mathrm{~nm}$ ShhN was added and live imaging was performed. Explants were fixed in $4 \%$ paraformaldehyde (PFA) for $15 \mathrm{~min}$ at room temperature for subsequent immunostaining.

Microscopy. Fluorescence and bright-field images were acquired with Leica DM6000 and DM4000 microscopes. Colocalization and in utero electroporation experiments were imaged with a Zeiss LSM 710 confocal microscope. Live-cell imaging was performed on a Leica DMIRE2 inverted microscope equipped with a heated environmental chamber $\left(37^{\circ} \mathrm{C}\right)$ 
and an MS-2000 XYZ automated stage (ASI). Time-lapse phase contrast images were acquired every 5 min using a $10 \times$ fluotar or $20 \times$ fluotar LD objective. All images were collected with an Orca ER CCD camera (Hamamatsu) using Volocity version 4.3 (Improvision).

Immunohistochemistry and histology. Immunohistochemistry was performed on $12-\mu \mathrm{m}$ thick cryosections. Primary antibodies used were against the following: Boc (R\&D Systems), neurofilament heavy chain 2H3 (Developmental Studies Hybridoma Bank), Tag1 4D7 (Developmental Studies Hybridoma Bank), Zic2 (a gift from S. Brown, University of Vermont, Burlington, VT) (Brown et al., 2003), EphB1 (Santa Cruz Biotechnology), Brn3b (Santa Cruz Biotechnology), L1 (Millipore), Cdon (R\&D Systems), and GFP (Abcam). We also generated a rabbit anti-Boc antibody directed against the cytoplasmic tail of mouse Boc. Immunofluorescence images were visualized using secondary antibodies conjugated to Alexa 488 or Alexa 568 (Invitrogen) and DAPI (Sigma). For DAB substrate immunostainings, sections were pretreated with $2 \%$ peroxide for 20 min to block endogenous peroxidase. The signal was detected using biotinylated secondary antibodies, $\mathrm{ABC}$ Vectastain Elite kit (Vector), and DAB substrate kit (Vector). Boc and Cdon expression was also detected in embryos heterozygous for the lacZ allele by using X-gal histochemistry (Okada et al., 2006). Histological analysis was performed on $12-\mu \mathrm{m}$-thick cryosections stained with hematoxylin and eosin.

Analysis of Boc mutant retina. The number of cells expressing RGC-specific markers was scored by averaging the number of positive cells in the VT retina from three E15 mouse retinal sections obtained from at least three embryos. The retinal areas quantified comprise a region of $200 \mu \mathrm{m}$ in the VT retina, measured from the ciliary body. For the quantification of the cell number in the ganglion cell layer (GCL) and developing inner nuclear layer (INL), we counted the cells within the respective layers of the VT area as observed by hematoxylin/eosin staining. For the developing INL, cells were distinguished based on their morphology and their specific location in the most basal part of the neuroblast layer (NBL).

Analysis of axonal retraction and growth cone collapse. For the analysis of axonal retraction using time-lapse imaging, only single axons that had grown $>200 \mu \mathrm{m}$ outside of the retinal explants were analyzed because we have shown using different markers that these long axons are all RGC axons. Axons that contacted a cell or another axon were excluded from the analysis. For growth cone collapse assays, growth cones were fixed 20 min after ShhN addition, by adding an equal volume of $8 \%$ PFA to the medium containing the retinal explants, at room temperature for $15 \mathrm{~min}$. RGCs were immunostained for Boc and with phalloidin (to label the growth cone). The length of axons, the level of fluorescence, and the area of growth cones were measured using Volocity.

Anterograde DiI labeling. Anterograde labeling of RGC axons was performed on fixed tissue using DiI as described previously (Plump et al., 2002). After DiI labeling, whole heads were sectioned at $30 \mu \mathrm{m}$. The sections were washed for $3 \mathrm{~min}$ in PBS and incubated for $10 \mathrm{~min}$ in DAPI at room temperature. The sections were then mounted in PBS and images taken immediately. For measurement of the relative ipsilateral projections, the area occupied by the optic nerve was selected and total DiI fluorescence was measured in this area. All images were acquired under conditions in which the pixels were not saturated. The data for each animal are an average of four consecutive frontal sections of the optic tracts just caudal to the optic chiasm. Measurements were taken blind to the genotype.

In utero electroporation. In utero electroporation was performed essentially as described previously (Garcia-Frigola et al., 2007). To coelectroporate Boc and YFP, DNA solutions were prepared with equal amounts of YFP expression plasmid $(2 \mu \mathrm{g} / \mu \mathrm{l})$ and pCAGGS-mouse Boc expression plasmid $(2 \mu \mathrm{g} / \mu \mathrm{l})$. Only the YFP plasmid was electroporated in the control conditions. The DNA solution was injected into the eye at E13.5 (in utero) and electroporated with 5 pulses ( $38 \mathrm{~V}, 50 \mathrm{~ms}$ ). Three days after electroporation (E16.5), the embryos were collected and their heads were dissected and fixed overnight at $4^{\circ} \mathrm{C}$ in $4 \%$ paraformaldehyde. Heads were then frozen and sectioned horizontally. Subsequent immunohistochemistry was performed to enhance YFP signal using a chicken anti-GFP antibody (Abcam).

Statistical analysis. Data were analyzed and graphs generated using Prism 4 statistical software (GraphPad Software). All error bars represent the SEM. Statistical significance was determined using twotailed Student's $t$ test, and values are as follows: ${ }^{\star} p<0.05,{ }^{* *} p<0.01$, ${ }^{* * *} p<0.001$.

\section{Results}

\section{Ipsilateral RGCs express the Shh receptor Boc}

We have previously shown that guidance of commissural axons by Shh requires the Shh receptor Boc (Okada et al., 2006). Because RGC axons also respond to Shh (Trousse et al., 2001; Kolpak et al., 2005; Sánchez-Camacho and Bovolenta, 2008), we 

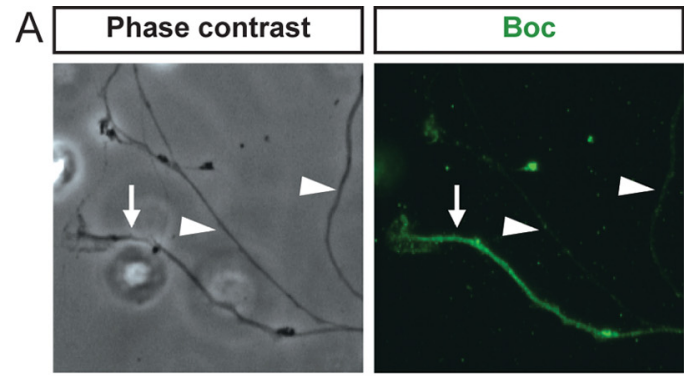

$\mathrm{B}$

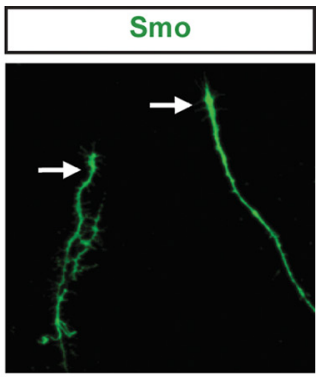

C
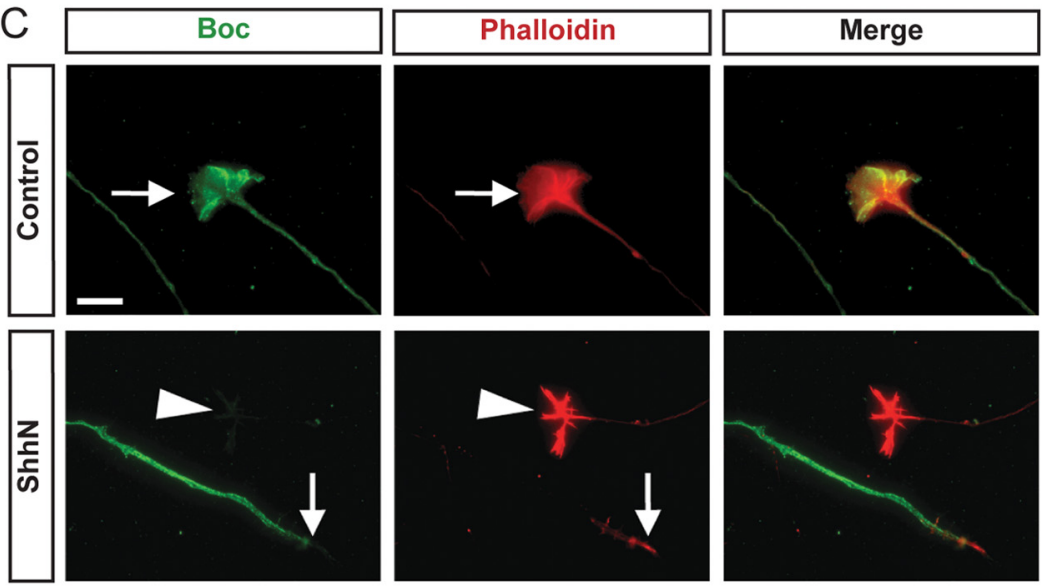

D

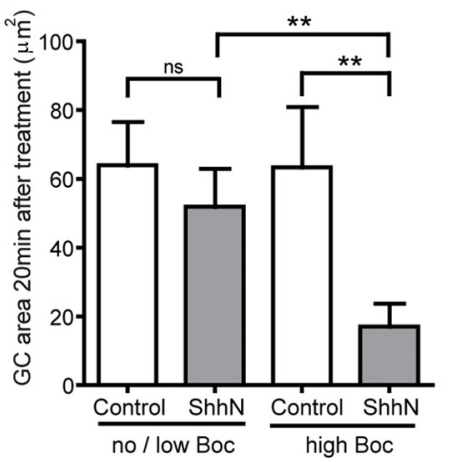

E

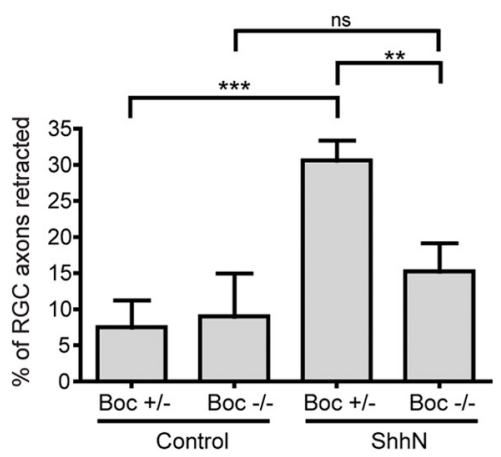

Figure 3. Shh specifically regulates axon and growth cone dynamics of Boc-expressing RGCs. $\boldsymbol{A}$, Immunofluorescent labeling of RGC axons from E17 rat retinal explants shows the presence of high Boc (rabbit anti-Boc antibody) levels in a subpopulation of RGC axons (arrow), while most of the axons exhibit no or low Boc levels (arrowhead). $\boldsymbol{B}$, Immunofluorescent labeling shows that the Shh signaling mediator Smoothened (Smo) is present on RGC axons. C, ShhN induces the growth cone collapse of RGC axons exhibiting high levels of Boc. Immunofluorescent labeling of Boc protein (green, rabbit anti-Boc antibody) in RGC growth cones (arrow), colabeled with the F-actin marker phalloidin (red) to delineate the morphology of the growth cone. Top, Growth cones of RGC axons expressing high levels of Boc remain uncollapsed 20 min after control treatment. Bottom, In contrast, 20 min after ShhN addition, RGC axons expressing a high level of Boc (arrow) have a collapsed growth cone, while the growth cones of RGC axons expressing no or low levels of Boc (arrowhead) are not sensitive to ShhN and do not collapse. Addition of control medium does not affect the size of RGC growth cones with a high level of Boc (top). D, Quantification of the collapse response from $\boldsymbol{C}$. Measurement of the size of the RGC growth cone area (in square micrometers) 20 min after treatment with control medium (white bars) or ShhN (gray bars) shows a significant reduction in the area of RGC growth cones that express a high level of Boc when treated with ShhN. In contrast, RGC growth cones with no/low Boc levels do not have a significant change in area in response to ShhN. $n=87$ growth cones. Error bars indicate SEM (one-way ANOVA with $p<0.05$ ). $\boldsymbol{E}$, Boc is required to mediate Shh-induced retraction of axons. Retinal explants isolated from $B \mathrm{CC}^{+/-}$and $B \mathrm{C}^{-/-} \mathrm{E} 15.5$ embryos were cultured for $24 \mathrm{~h}$. RGC axon dynamics were analyzed by time-lapse microscopy after addition of $10 \mathrm{~nm}$ ShhN. The percentage of RGC axons exhibiting net retraction 90 min after ShhN treatment was significantly higher for $\mathrm{Boc}^{+/-}$RGCs treated with ShhN (30.6\%) than for those treated with control medium (7.5\%, $p=0.0002$ ), while $B o c^{-1-}$ RGC axons treated with ShhN were not significantly different from $B o c^{-1-}$ RGCs treated with control medium ( $p>0.05$ ) (one-way ANOVA with Bonferonni's post-test, ns $=p>0.05,{ }^{* *} p<0.01$, $\left.{ }^{* * *} p<0.001\right)$. Error bars indicate SEM. Scale bar, $10 \mu \mathrm{m}$. hypothesized that this response might also occur via Boc, so we first assessed whether RGCs express Boc.

We characterized Boc expression using our mice harboring a lac $Z$ reporter gene targeted to the Boc locus ( $b o c^{\text {lacZ/+ }}$ mice) (Okada et al., 2006). Using $\beta$-galactosidase activity histochemistry on sections of developing retina from E15.5 mouse embryos, we detected a strong enrichment of Boc expression in the VT crescent of the retina (Fig. 1A). This expression was present in the GCL and the NBL of the retina, suggesting expression in both RGCs and retinal progenitors, respectively (Fig. $1 A^{\prime}, A^{\prime \prime}$ ). We also detected Boc expression in the ciliary bodies, which are neurogenic niches located at the periphery of the retina. Cdon, another Shh receptor closely related to Boc (Okada et al., 2006), is also expressed in RGCs but, in contrast to Boc, the Cdon-positive cells were distributed throughout the entire retina and did not show VT enrichment (Fig. S1, available at www.jneurosci.org as supplemental material).

We next assessed Boc protein expression by immunohistochemistry. In agreement with the reporter gene analysis, we found that Boc protein is highly enriched in the VT crescent of the retina (Fig. $1 B-$ $\left.B^{\prime \prime}\right)$. Boc protein appears to be preferentially located in the GCL of the retina, suggesting that it is mostly present in RGCs. Using later stage rat E17.5 retina sections, we confirmed that Boc is strongly expressed in the GCL, in which RGC somas are located, and in the RGC axon layer (RAL), in which RGC axons are located (Fig. 1C). The axonal localization of Boc was further confirmed by colocalization with neurofilament, a specific marker of RGC axonal projections in the retina (Fig. $1 C^{\prime \prime \prime}$ ). Together, these results indicate that Boc is expressed by VT RGCs.

Contrary to other RGCs of the retina, the majority of RGCs of the VT crescent do not cross the optic chiasm and, instead, project ipsilaterally (Petros et al., 2008). Hence, the highly restricted expression of Boc to RGCs of the VT crescent suggested that Boc might be expressed in RGCs that project ipsilaterally. To test this, we performed colocalization studies of Boc and Zic2, a nuclear marker of ipsilateral RGCs (Herrera et al., 2003). We observed an enrichment of Zic2 in the VT retina and a strong colocalization with Boc-positive cells (Fig. 1D,E). EphB1, a receptor expressed by ipsilateral RGCs and important for their repulsion away from the optic chiasm (Williams et al., 2003), also colocalized with Boc, both in RGC soma 
and axons (Fig. S2, available at www. jneurosci.org as supplemental material). Together, these results indicate that Boc is enriched in VT RGCs and that ipsilateral RGCs express Boc.

\section{Shh specifically regulates axon and growth cone dynamics of Boc-expressing RGCs}

Shh causes the retraction of RGC axons in vitro (Trousse et al., 2001; Kolpak et al., 2005; Sánchez-Camacho and Bovolenta, 2008) and it is present in the chiasmatic zone (Torres et al., 1996; Marcus et al., 1999; Wallace and Raff, 1999; Trousse et al., 2001). Based on these data and on our finding that Boc is expressed in ipsilateral RGCs, we hypothesized that Boc might function as a Shh receptor which prevents these axons from crossing the optic chiasm. We thus investigated whether Shh could control the behavior of ipsilateral RGC axons.

Many of the experiments implicating a role for Shh in the guidance of RGC axons were performed over a long time period $(24-48 \mathrm{~h})$ and therefore did not distinguish between short-term, direct effects and long-term, indirect effects of Shh on the growth cone of these axons (Trousse et al., 2001; Kolpak et al., 2005; SánchezCamacho and Bovolenta, 2008). Moreover, it is not clear whether Shh has a direct effect on all RGC axons, or only on a subpopulation of RGCs. To address this, we cultured retinal explants and performed time-lapse analysis of RGC axon behavior in response to Shh. Using markers of RGC axons, we confirmed that the long projections growing out of RGC explants were exclusively RGC axons (Fig. S3, available at www. jneurosci.org as supplemental material). In basal conditions (in the absence of Shh), most RGC axons exhibited net axonal growth over a period of $90 \mathrm{~min}$, with only $14.0 \pm 3.3 \%$ of axons exhibiting a net retraction during this period (Fig. 2). This reflects the normal dynamics of axon growth and retraction also observed with other neuron types (Yam et al., 2009). Addition of 10 $\mathrm{nM}$ ShhN, a concentration previously shown to induce turning of commissural axons (Yam et al., 2009), led to a significant increase in the number of axons exhibiting net retraction over a $90 \mathrm{~min}$ period, with an almost threefold increase to $38.5 \pm 3.4 \%$ of axons retracting (Fig. 2 E; Fig. S4, available at www.jneurosci.org as supplemental material). These results indicate that only a subset [comprising $24.5 \%$ (38.5\% minus $14.0 \%$ )] of RGC axons retract in response to Shh. Of the neurons which responded to Shh, we observed that addition of Shh caused their growth cones to collapse and their axons to rapidly begin to retract, with a median time to initiate retraction of $15 \mathrm{~min}$ (Fig. 2D). Addition of SANT-1, a specific Smo inhibitor (Chen et al., 2002), completely blocked the effect of Shh (Fig. 2E), indicating that Shh-induced retraction requires $S m o$ activity.

We next tested whether the subset of RGC axons that retract in response to Shh corresponds to a specific and identifiable sub- population of RGCs. Since Boc is expressed in ipsilateral RGCs (Fig. 1) and has been implicated in the guidance of commissural axons, we assessed whether the RGCs responding to Shh correspond to a Boc-expressing subpopulation.

Immunofluorescent staining of RGC axons showed that Boc and Smo proteins are present in the axon and the growth cone of RGCs (Fig. 3A-C), similar to commissural neurons (Okada et al., 2006). Consistent with the restricted expression of Boc observed on retina sections (Fig. 1), we found that only a subpopulation (24.8\%) of RGC axons express Boc in vitro (Fig. $3 \mathrm{~A}, \mathrm{C}$ ), while the other RGCs expressed undetectable (or very low) levels of Boc (thereafter referred to as "no/low Boc"). Of interest, the percentage of RGC axons expressing high levels of Boc was similar to the percentage of axons responding to Shh in vitro (24.8\% versus $24.5 \%$, respectively), raising the possibility that they might correspond to the same population.

To directly test whether the RGCs expressing Boc might be the ones responding to Shh in vitro, we compared the growth cone collapse activity of Shh on no/low Boc growth cones versus growth cones expressing high Boc levels. This was performed 20 min after the addition of Shh, at an early phase of the response when the growth cones had already collapsed but the axons were only beginning to retract. Only the growth cones with high level 
A
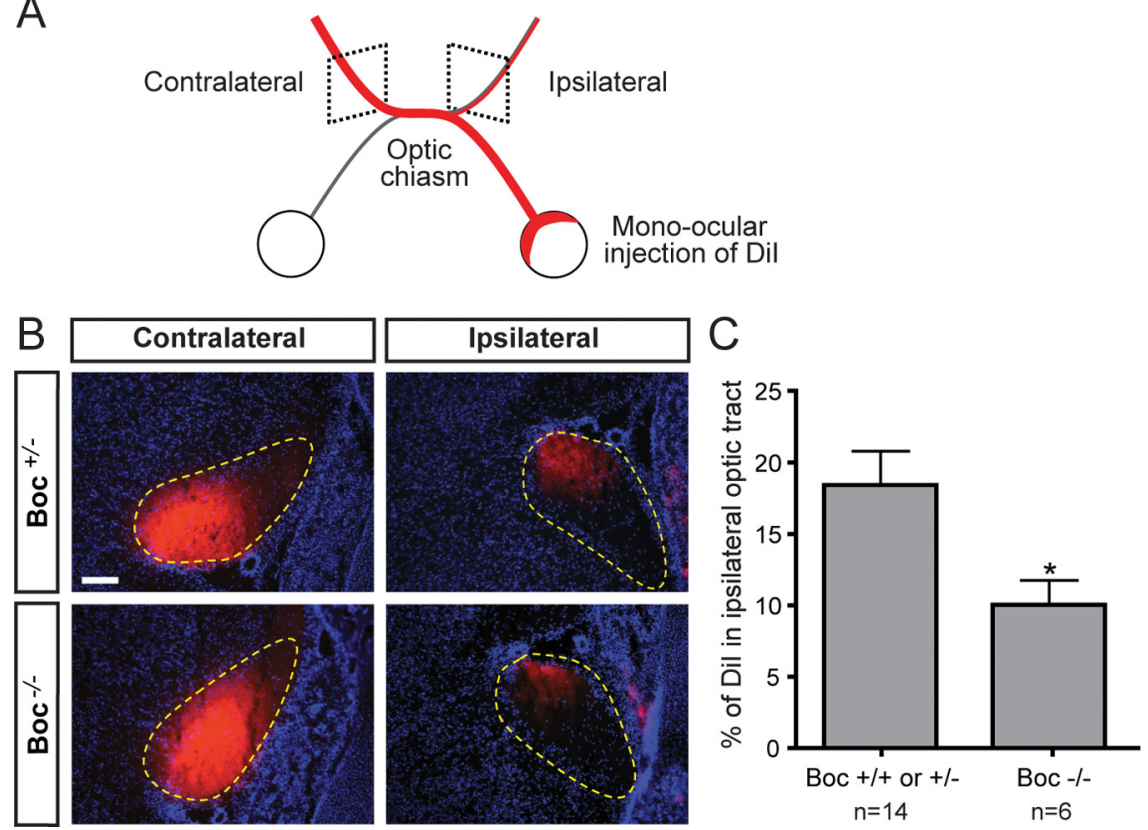

Figure 5. Normal segregation of ipsilateral axons at the optic chiasm requires Boc. $A$, Schematic of the rostral part of the E18.5 mouse visual system after mono-ocular injection of Dil crystals (red) in the whole retina. $B$, Coronal sections of $E 18.5 \mathrm{Boc}^{+/-}$and $B \mathrm{C}^{-1-}$ optic tracts after mono-ocular injection of Dil crystals (red) and counterstaining with DAPI (blue). The optic tracts are circled with yellow dashed lines. The Dil intensity is lower in the ipsilateral tract of $\mathrm{BOC}^{-/-}$compared with $\mathrm{Boc}^{+/-}$mice. Note that although these images show saturated pixels for ease of viewing the faint ipsilateral signal, the quantification was performed on unsaturated images. C, Quantification of the percentage of Dil signal in the ipsilateral optic tract as a proportion of the total Dil signal from the contralateral and ipsilateral optic tract reveals a significant decrease in ipsilateral projections in $\mathrm{Boc}^{-/-}$mice compared with control $\left(\mathrm{Boc}^{+/-}\right.$or $\left.\mathrm{Boc}^{+/+}\right)$mice $[18.5 \pm 2.5 \%(n=14$ embryos) and $10.0 \pm 1.8 \%(n=6)$, respectively; $p<$ 0.05 using Student's unpaired $t$ test]. Scale bar, $100 \mu \mathrm{m}$.

of Boc collapsed following Shh addition, while nearby growth cones not expressing Boc did not collapse (Fig. 3C,D; Fig. S5, available at www.jneurosci.org as supplemental material). Hence, Boc-expressing RGCs collapse in response to Shh, but RGCs not expressing Boc do not.

To test whether Boc is required to mediate the response of RGCs to Shh, we performed Shh retraction assays on mouse retinal explants isolated from Boc mutant embryos (Okada et al., 2006). In the absence of Shh, RGC axons in explants from Boc heterozygous and Boc mutant embryos had the same basal percentage of retracted axons after $90 \mathrm{~min}$ (Fig. 3E), indicating that Boc is not required for their Shh-independent dynamics. Upon addition of Shh, RGC axons from Boc heterozygous explants had a significant increase in retraction compared with the control group (30.6 $\pm 2.6 \%$ versus $7.5 \pm 3.8 \%$, respectively, $p<0.001)$. However, RGC axons from Boc mutant explants did not show a significant increase in their retraction compared with the control untreated Boc mutant explant group ( $p=0.399)$. Together, these results indicate that the Boc-expressing RGCs are the RGCs responsive to Shh and that Boc is required in these neurons to mediate their retraction response to Shh.

\section{Boc mutant mice have normal retinal cell fate}

Our in vitro results raised the possibility that Boc might play a role in the guidance of ipsilateral RGC axons in vivo. However, since Shh also controls the proliferation and differentiation of RGCs (Jensen and Wallace, 1997; Zhang and Yang, 2001; Lupo et al., 2005; Wang et al., 2005; Locker et al., 2006), it was important to first assess whether Boc mutant retinas are generated normally, before analyzing Boc-dependent axon guid- ance in these animals. To address this, we analyzed layer formation and cell fate specification in Boc mutant retina (Fig. 4). We counted the number of cells in the GCL and in the INL. No difference was observed between wild-type, $\mathrm{Boc}^{+/-}$, and $\mathrm{Boc}^{-/-}$retinas (Fig. $4 D, E$ ). We used multiple RGC markers, including two general RGC markers (Brn3b and L1) and two ipsilateral-specific RGC markers (Zic2 and EphB1), to assess the integrity of RGC cell fate specification in Boc mutant retina. We also looked at the expression of Cdon, which is expressed in the majority of RGCs. All the markers analyzed were expressed in a similar pattern and at similar levels in $\mathrm{Boc}^{+/-}$and $\mathrm{Boc}^{-/-}$retina (Fig. $4 \mathrm{~A}$ ). Counting the number of RGCs (using Brn3b as a marker) present in the VT did not reveal any difference between $\mathrm{Boc}^{+/-}$ and $\mathrm{Boc}^{-/-}$retinas (Fig. $4 \mathrm{C}$ ). Importantly, the number of Zic2-positive ipsilateral RGCs generated was the same in wild-type, $\mathrm{Boc}^{+/-}$, and $\mathrm{Boc}^{-/-}$retinas (Fig. $4 \mathrm{~B}$ ). Thus, Boc mutant retinas have no apparent defects in layer formation, RGC number, and RGC fate specification, indicating that the growth and differentiation effects of Shh in the retina are not mediated by Boc.

\section{Normal segregation of ipsilateral axons} at the optic chiasm requires Boc

If Boc is required in vivo for ipsilaterally projecting RGC axons to respond to Shh present at the diencephalon midline, it would be predicted that the ipsilateral projections of Boc mutant mice would have pathfinding defects. To test whether Boc is required in vivo for the guidance of ipsilaterally projecting RGC axons at the chiasm, we performed complete mono-ocular DiI-filling of the retina of E18 mouse embryos to estimate the relative proportion of axons entering the contralateral versus the ipsilateral optic tract (Plump et al., 2002; Williams et al., 2003). After allowing the dye to diffuse, we cut frontal sections caudal to the optic chiasm, and quantified the amount of DiI fluorescence signal in the contralateral and ipsilateral optic tracts (Fig. $5 A, B$ ). This analysis revealed that the percentage of ipsilateral axon signal [calculated by dividing the ipsilateral axon signal over the total signal (ipsilateral plus contralateral)] in mice heterozygous and wild-type for Boc was $18.5 \pm 2.5 \%$ (Fig. $5 C$ ), consistent with values previously determined by others using the same technique (17\%) (Williams et al., 2003). However, in mice homozygous for the Boc mutation, the percentage of ipsilateral axon signal was strongly reduced with only $10.0 \pm 1.8 \%$ of the DiI signal in the ipsilateral optic tract. These results indicate that the lack of Boc leads to a reduction in the number of axons in the ipsilateral optic tract and provide evidence that Boc is required in vivo for the normal segregation of ipsilateral projections at the optic chiasm.

Ectopic Boc expression in contralateral RGCs prevents their axons from crossing the optic chiasm

We next asked whether Boc expression is sufficient to determine RGC axon choice at the optic chiasm. Using in utero electroporation, we introduced vectors encoding either yellow fluorescent 
protein (YFP) or a combination of Boc and YFP in RGCs at E13.5, just before the peak phase of RGC axon growth (Petros et al., 2008). We analyzed the trajectories of RGC axons at the optic chiasm at E16.5, a time when most RGC axons from the peak phase have projected beyond the chiasm. Using this approach, only the nasal retina was targeted for electroporation, leading to expression exclusively in contralaterally projecting RGCs (Fig. 6B), and their axons could be followed far past the chiasm in the optic tract (Garcia-Frigola et al., 2007). Axons expressing YFP alone projected contralaterally and only very rarely entered the ipsilateral optic tract or contralateral optic nerve (Fig. 6D-F).

When YFP was coexpressed with Boc, two types of defects, both consistent with a role for Boc in preventing contralateral RGC axons from crossing the optic chiasm, were observed. First, we observed that many Boc-electroporated axons failed to pass the chiasm; instead these axons re-entered in the contralateral optic nerve toward the eye (Fig. $6 F^{\prime}$ ). This phenotype was observed in almost all (93\%) of the Boc-electroporated embryos and represented $9.3 \pm 1.8 \%$ of all Bocelectroporated RGC axons, compared with $2.5 \pm 1.0 \%$ in YFP controls (Fig. 6 F). In these experiments, Boc expression in presumptive contralaterally projecting RGCs appears to render them sensitive to Shhmediated repulsion, thus making their axons avoid the chiasm and enter the contralateral optic nerve.

The second type of defect that we observed in Boc-electroporated axons was an increase in ipsilateral projections at the chiasm. In the YFP control condition, $1.8 \pm 0.4 \%$ of the total number of axons projected to the ipsilateral optic tract (Fig. $6 E)$. When Boc was electroporated, we observed a fourfold increase in the percentage of ipsilateral axons, with an average of $7.2 \pm 1.9 \%$ exhibiting this behavior (Fig. $6 E^{\prime}$ ), indicating that Boc expression in contralateral RGCs prevents their axons from crossing the optic chiasm, and they instead project ipsilaterally. This phenotype was observed in $67 \%$ of the Boc-electroporated embryos. Of note, a similar increase in ipsilaterally projecting axons has been observed in EphB2 and EphB1 gain of function experiments, with three- and sixfold increases, respectively, when EphB2 or EphB1 are ectopically expressed in contralaterally projecting RGCs, compared with control (Petros et al., 2009). These results suggest that, similarly to EphB1 and EphB2, Boc expres-

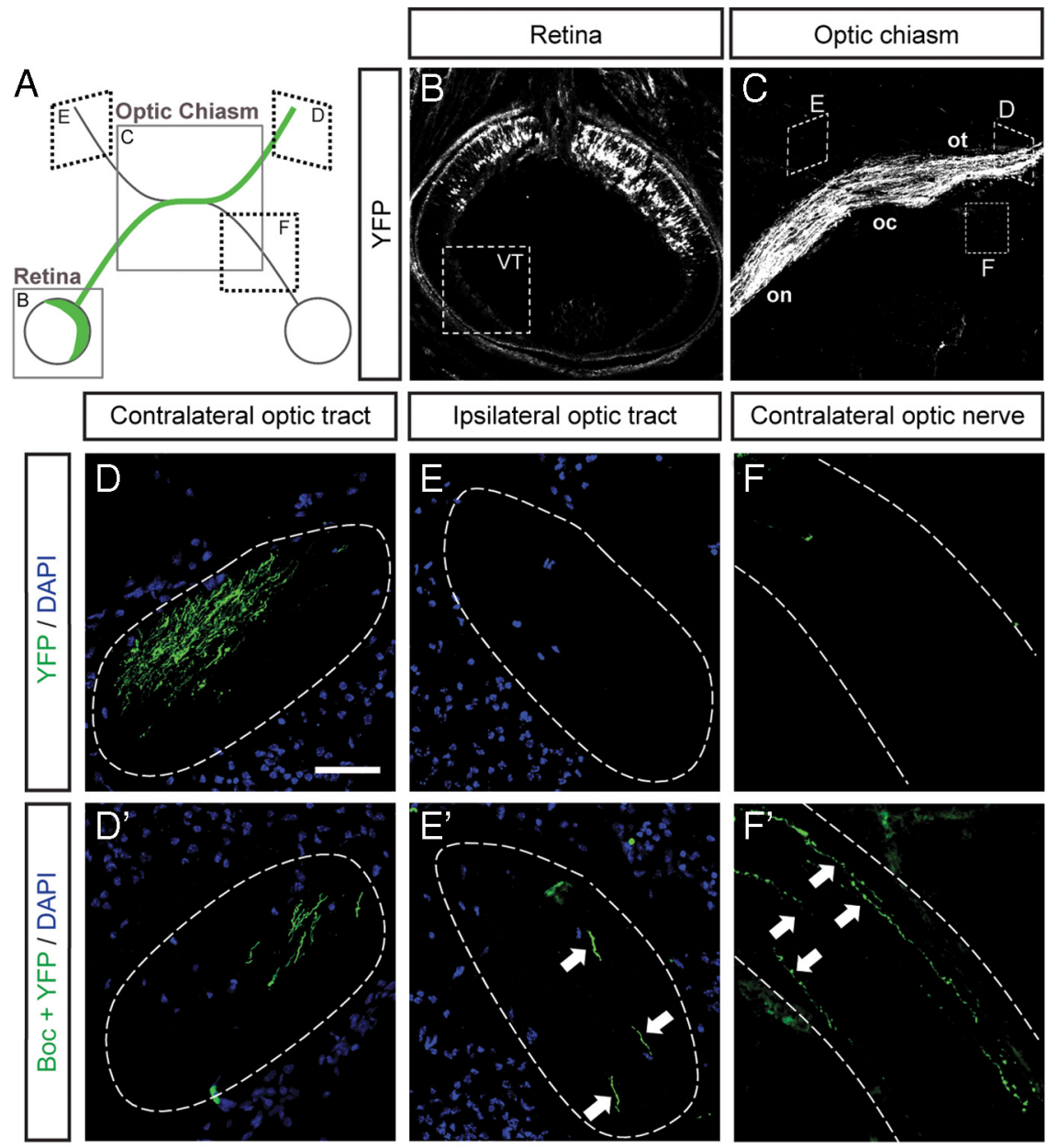

$E^{\prime \prime}$

F"
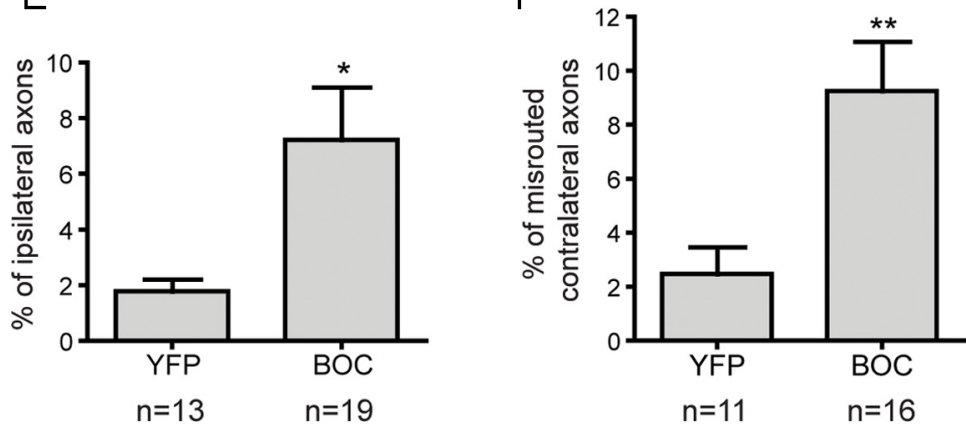

Figure 6. Ectopic Boc expression in contralateral RGCs prevents their axons from crossing the optic chiasm. $A$, Schematic of the rostral part of the E16.5 mouse visual system, $3 \mathrm{~d}$ after in utero electroporation of the retina at E13.5. The positions of the retina $(\boldsymbol{B})$ and the optic chiasm $(\boldsymbol{C})$ are indicated by the boxes. The green line indicates the normal path of YFPelectroporated contralateral RGC axons. $\boldsymbol{B}$, Horizontal section of an E16.5 mouse retina $3 \mathrm{~d}$ after electroporation. Due to electrode positioning, the VT region never contained YFP-positive cells. $C$, Control axons expressing YFP alone exhibit normal axon pathfinding, crossing the optic chiasm and reaching the contralateral optic tract $(\boldsymbol{D})$. They very rarely enter the ipsilateral optic tract $(\boldsymbol{E})\left(1.8 \pm 0.4 \%, \boldsymbol{E}^{\prime \prime}\right)$ or the contralateral optic nerve $(\boldsymbol{F})\left(2.5 \pm 1.0 \%, \boldsymbol{F}^{\prime \prime}\right) . \boldsymbol{D}, \boldsymbol{D}^{\prime}$, Semiperpendicular sections of the contralateral optic tract. $\boldsymbol{E}, \boldsymbol{E}^{\prime}$, Semiperpendicular sections of the ipsilateral optic tract. $\boldsymbol{F}, \boldsymbol{F}^{\prime}$, Parallel sections of the contralateral optic nerve. When Boc is coelectroporated with YFP (Boc +YFP), Boc + YFP-expressing axons enter the ipsilateral optic tract $\left(\boldsymbol{E}^{\prime}\right)$ and contralateral optic nerve $\left(\boldsymbol{F}^{\prime}\right)$ at a higher frequency. $\boldsymbol{E}^{\prime \prime}, \boldsymbol{F}^{\prime \prime}$, Quantification of the percentage of misguided RGC axons over the total number of electroporated axons. $\boldsymbol{E}^{\prime \prime}$, Histogram showing that the percentage of ipsilateral axons is significantly increased (by fourfold, $p=0.0262$ using Student's unpaired $t$ test) when RGCs express Boc + YFP compared with YFP alone (YFP, $n=13 ;$ Boc, $n=19$ embryos). $\boldsymbol{F}^{\prime \prime}$, Histogram showing that the percentage of axons re-entering the contralateral optic nerve is significantly higher (by 3.7-fold, $p=0.0085$ using Student's unpaired $t$ test) in RGCs expressing Boc + YFP compared with YFP alone (YFP, $n=11 ;$ Boc + YFP, $n=16$ embryos). on, Optic nerve; oc, optic chiasm; ot, optic tract. Scale bar, $100 \mu \mathrm{m}$. 
sion is sufficient to reroute contralaterally projecting into ipsilaterally projecting RGC axons at the chiasm.

\section{Discussion}

We have previously shown that Boc, a Shh receptor which has an extracellular domain architecture homologous to other guidance receptors such as Robos and DCC, is required for the guidance of commissural axons by Shh (Okada et al., 2006). In this paper, we present evidence supporting a role for Boc in mediating the guidance effect of Shh on ipsilateral-projecting RGCs. First, we found that Boc is expressed in $\mathrm{Zic} 2{ }^{+}$and EphB1 ${ }^{+}$RGCs, confirming that Boc is expressed in ipsilateral RGCs. Second, to test whether the $\mathrm{Boc}^{+}$subpopulation of RGCs responds to Shh, we cultured retinal explants and found that only Bocpositive growth cones collapsed in response to Shh. Third, using our RGC retraction assay, we found that Shh-mediated retraction requires Boc, as the response is lost in Boc mutant RGCs. That Shh-mediated growth cone collapse and retraction occur rapidly (within $20 \mathrm{~min}$ ) supports a direct effect of Shh on these axons. In vivo, using anterograde labeling of RGC axons in Boc mutant mice, we show that Boc is required for the normal segregation of ipsilateral axons in the optic chiasm (Fig. 7), and conversely, we show that Boc expression in presumptive contralateral RGCs induces misrouting of their axons at the optic chiasm, suggesting that Boc is sufficient to confer Shh sensitivity to these axons. Together, these results suggest that Shh repels ipsilateral RGC axons at the optic chiasm via its receptor Boc. This work identifies a novel molecular pathway required for the segregation of ipsilateral axons at the optic chiasm.

\section{The roles of Shh in the guidance of RGC axons}

Previous studies have supported a role for Shh in the guidance of RGC axons. Earlier work in chick proposed that Shh, which is expressed in the diencephalic ventral midline, acts as a repellent to guide RGC axons through the optic chiasm (Trousse et al., 2001). Recently, it has been shown that Shh has different effects on the outgrowth and growth cone size of RGC neurites depending on their origin (dorsonasal vs VT), with Shh promoting the growth of VT retinal explants over 24-48 h (Sánchez-Camacho and Bovolenta, 2008). Our in vitro experiments show that the ability of RGCs to respond to Shh in the short term depends on the expression of Boc, and it remains to be determined whether the longer-term increase in outgrowth previously observed in VT-derived neurites also depends on Boc.

In addition to in vitro experiments, in vivo experiments have also suggested a role for Shh in mouse RGC axon pathfinding (Sánchez-Camacho and Bovolenta, 2008). Injection of E13.5 mouse embryos with an hybridoma producing a Shh-blocking antibody (and analyzed at E18.5) alters the distribution of RGC axons at the optic chiasm with an enlargement of the overall region occupied by RGC fibers at the midline and axon extension in aberrant directions. However, as Shh activity is important to pattern the midbrain regions surrounding the ventral hypothalamus (just adjacent to the optic chiasm) during retinal axon development (Dale et al., 1997; Barresi et al., 2005; Manning et al., 2006), it is likely that blocking Shh signaling for $5 \mathrm{~d}$ in developing embryos can cause patterning defects. Thus, as noted by the authors (Sánchez-Camacho and Bovolenta, 2008), it is possible that the effects observed are not due to a direct guidance effect of Shh but rather to changes in expression of other guidance cues. In a second set of experiments, an inhibitor of the Shh signaling mediator Smo was electroporated in the developing mouse visual

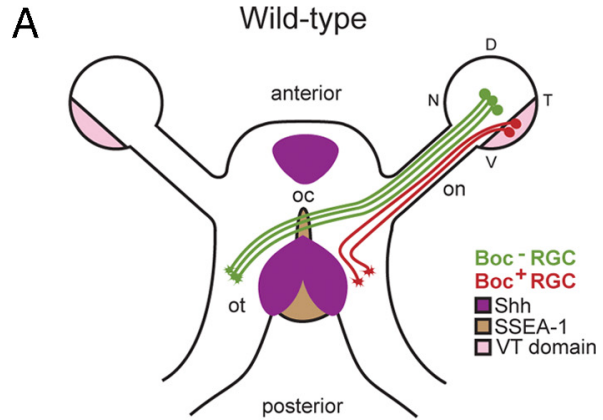

$\mathrm{B}$

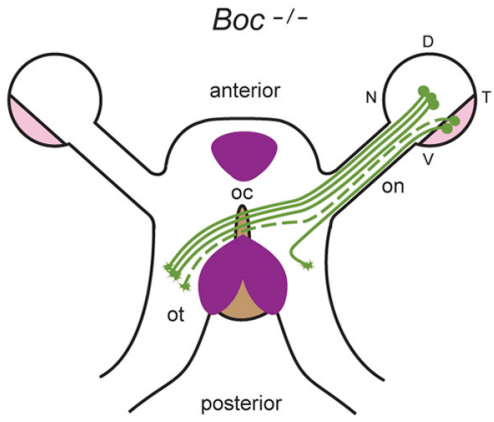

C Ectopic Boc expression in contralaterally projecting neurons

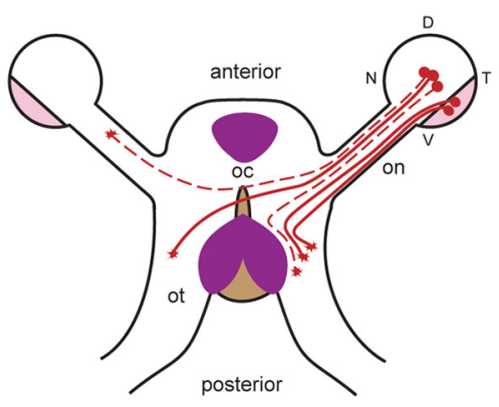

Figure 7. Summary of the Boc gain- and loss-of-function phenotypes at the chiasm. $A$, In wild-type conditions, axons expressing Boc (red) originate from the VT retina and avoid Shh at the optic chiasm. $\boldsymbol{B}$, In Boc mutant mice, VT axons do not express Boc and fail to detect Shh at the midline. Instead of being repelled by Shh, they cross the chiasm and project contralaterally (represented by the dashed line). C, Ectopic expression of Boc in presumptive contralateral RGCS prevents their axons from crossing the optic chiasm. Two types of defects were observed: the conversion of contralateral projections into ipsilateral projections and the misrouting of axons to the contralateral optic nerve (represented by the dashed lines). V, Ventral; D, dorsal; $\mathrm{N}$, nasal; T, temporal; on, optic nerve; oc, optic chiasm; ot, optic tract.

system at E13.5 and analyzed at E16.5 (Sánchez-Camacho and Bovolenta, 2008). Electroporated presumptive contralaterally projecting RGC axons exhibited growth and guidance defects, suggesting that Shh functions cell-autonomously to control the pathfinding of these axons. However, this experiment did not assess whether ipsilaterally projecting RGC axons are guided normally at the chiasm in these conditions and, therefore, whether Shh signaling guides ipsilateral projections in vivo remained unknown until now.

Our in vivo data indicate that Boc is required for the normal segregation of ipsilateral axons in the optic chiasm, but not for RGC fate specification. Although we have not shown that, in vivo, the role of Boc is dependent on its ability to bind Shh, based on our in vitro experiments showing that Boc is required for the axonal response of RGCs to Shh, we propose that Boc functions as a Shh receptor to prevent ipsilateral RGC axons from crossing the optic chiasm. 
In contrast to Sánchez-Camacho and Bovolenta, we found that, in vitro, only Boc-positive RGC axons retract in response to Shh and that this response is dependent on Boc. Additionally, Sánchez-Camacho and Bovolenta did not observe an induction of VT explant growth cone collapse by Shh. One possibility for this discrepancy is that they did not look specifically at ipsilaterally projecting RGCs. Additionally, differences in the methods used to quantify growth cone collapse, the origin of the retinal tissue (mouse E14.5 versus rat E17), the concentration of laminin, and the concentration of Shh used to induce collapse might explain the difference between the results, with the laminin concentration and Shh concentration both potentially affecting the sensitivity of the cells to collapse in response to Shh. Although we did not see any effect of Shh on Boc-negative RGCs in vitro, our results do not exclude a role for Shh-mediated guidance of contralaterally projecting RGCs in vivo (Sánchez-Camacho and Bovolenta, 2008) or that Shh might have longer term growth effects on contralaterally projecting RGCs. However, as Boc is not expressed in these RGCs, the identity of the Shh receptor mediating this effect of Shh on contralaterally projecting axons remains to be determined.

\section{Boc is a bifunctional axon guidance receptor for Shh}

We have previously shown that Boc is required for the attraction of spinal cord commissural axons by Shh (Okada et al., 2006). Here, our data show that Boc is required for the retraction response of ipsilateral RGC axons to Shh. Thus, Boc is required to mediate both positive (attraction) and negative (retraction) guidance effects of Shh. This is similar to other axon guidance receptors, which can also mediate positive and negative effects. The opposite effects of Shh on commissural and RGC axons might be due to modulation of the response by an intrinsic and/or extrinsic factor. For example, extrinsic factors can convert Netrin attraction to repulsion by modulating cyclic nucleotide levels (Höpker et al., 1999). Alternatively, it is also possible that these two effects are mediated by distinct Boc receptor complexes that result in opposite guidance effects - a possibility that also has a precedent in the case of Netrin/UNC-6, which attracts axons by activating DCC/UNC-40 family receptors, but becomes a repellent when UNC5 h/UNC-5 family receptors are present (Hamelin et al., 1993; Hong et al., 1999). Further experiments will be required to determine the molecular mechanism underlying this differential effect of Shh on commissural and RGC axons.

\section{A novel molecular pathway required for the segregation of ipsilateral RGC axons at the optic chiasm}

Although other molecules in addition to EphBs/ephrin-Bs have been shown to play a role in the guidance of RGC axons, none of them have been shown to control the ipsilateral guidance decision of RGCs (Petros et al., 2008). For example, in vitro, contralaterally and ipsilaterally projecting axons do not respond differentially to inhibition of EphA signaling (as they do for EphB signaling) (Marcus et al., 2000) and, in vivo, no evidence for abnormal chiasm guidance have been observed in Ephrin-A mutant mice (Feldheim et al., 2000; Haustead et al., 2008). Additionally, the Slit proteins, through their Robo receptors, channel RGC axons into their normal paths and regulate their fasciculation, but normal ipsilateral projections form in the Slit1;Slit2 mutant mice (Plump et al., 2002). Finally, Nr-CAM mediates midline crossing of contralateral axons, but does not affect the guidance of ipsilateral axons (Williams et al., 2006). Since the EphBs/ephrin-Bs account for the pathfinding of only half of the ipsilateral projec- tions, other factors which regulate ipsilateral segregation remained to be identified.

Our results indicate that, similarly to EphB receptors, Boc is required in vivo for the proper segregation of ipsilateral projections at the optic chiasm. Importantly, this effect is not due to a decrease in the number of ipsilateral RGCs generated or to the loss of EphB1 receptor expression. Therefore, we propose that signaling through the Boc receptor is a novel molecular mechanism required for the segregation of ipsilateral RGC axons at the optic chiasm.

We show that the Boc knock-out has an $\sim 50 \%$ reduction in the size of the ipsilateral optic tract. This indicates that Boc is not required for all of the ipsilateral axons to segregate at the optic chiasm. Similarly, a partial phenotype was also observed in the EphB1;EphB2;EphB3 triple knock-out (Williams et al., 2003). These observations are consistent with two models. In the first model, EphBs and Boc could function in two different subpopulations of ipsilateral RGCs and each receptor could account independently for the ipsilateral segregation of their subpopulation. In this model, the $50 \%$ of ipsilateral axons still projecting normally in the Boc mutant mice would be an EphB-dependent subpopulation, and vice versa in the EphBs mutant mice. In the second model, EphBs and Boc could both function in the whole population of ipsilateral RGCs and play partially redundant roles. In this model, the $50 \%$ of ipsilateral axons still projecting normally in the Boc mutant mice would be guided by partially redundant EphB-dependent guidance, and vice versa in the EphBs mutant mice. Since we observed that most EphB $1^{+}$RGCs express Boc, we favor the second model. Regardless of whether Boc and EphBs function in the same ipsilateral RGCs or in two different subpopulation of RGCs, it will be interesting to see whether the loss of both types of receptors (Boc and EphBs) result in the complete absence of ipsilateral projections.

\section{References}

Barresi MJ, Hutson LD, Chien CB, Karlstrom RO (2005) Hedgehog regulated Slit expression determines commissure and glial cell position in the zebrafish forebrain. Development 132:3643-3656.

Brown LY, Kottmann AH, Brown S (2003) Immunolocalization of Zic2 expression in the developing mouse forebrain. Gene Expr Patterns 3:361-367.

Charron F, Tessier-Lavigne M (2005) Novel brain wiring functions for classical morphogens: a role as graded positional cues in axon guidance. Development 132:2251-2262.

Charron F, Stein E, Jeong J, McMahon AP, Tessier-Lavigne M (2003) The morphogen sonic hedgehog is an axonal chemoattractant that collaborates with netrin-1 in midline axon guidance. Cell 113:11-23.

Chen JK, Taipale J, Young KE, Maiti T, Beachy PA (2002) Small molecule modulation of Smoothened activity. Proc Natl Acad Sci USA 99:14071-14076.

Dale JK, Vesque C, Lints TJ, Sampath TK, Furley A, Dodd J, Placzek M (1997) Cooperation of BMP7 and SHH in the induction of forebrain ventral midline cells by prechordal mesoderm. Cell 90:257-269.

Erskine L, Herrera E (2007) The retinal ganglion cell axon's journey: insights into molecular mechanisms of axon guidance. Dev Biol 308:1-14.

Feldheim DA, Kim YI, Bergemann AD, Frisén J, Barbacid M, Flanagan JG (2000) Genetic analysis of ephrin-A2 and ephrin-A5 shows their requirement in multiple aspects of retinocollicular mapping. Neuron 25:563-574.

Garcia-Frigola C, Carreres MI, Vegar C, Herrera E (2007) Gene delivery into mouse retinal ganglion cells by in utero electroporation. BMC Dev Biol 7:103.

García-Frigola C, Carreres MI, Vegar C, Mason C, Herrera E (2008) Zic2 promotes axonal divergence at the optic chiasm midline by EphB1dependent and -independent mechanisms. Development 135:1833-1841. Hamelin M, Zhou Y, Su MW, Scott IM, Culotti JG (1993) Expression of the 
UNC-5 guidance receptor in the touch neurons of $C$. elegans steers their axons dorsally. Nature 364:327-330.

Haustead DJ, Lukehurst SS, Clutton GT, Bartlett CA, Dunlop SA, Arrese CA, Sherrard RM, Rodger J (2008) Functional topography and integration of the contralateral and ipsilateral retinocollicular projections of Ephrin$A^{-1-}$ mice. J Neurosci 28:7376-7386.

Herrera E, Brown L, Aruga J, Rachel RA, Dolen G, Mikoshiba K, Brown S, Mason CA (2003) Zic2 patterns binocular vision by specifying the uncrossed retinal projection. Cell 114:545-557.

Hong K, Hinck L, Nishiyama M, Poo MM, Tessier-Lavigne M, Stein E (1999) A ligand-gated association between cytoplasmic domains of UNC5 and DCC family receptors converts netrin-induced growth cone attraction to repulsion. Cell 97:927-941.

Höpker VH, Shewan D, Tessier-Lavigne M, Poo M, Holt C (1999) Growthcone attraction to netrin-1 is converted to repulsion by laminin-1. Nature 401:69-73.

Jensen AM, Wallace VA (1997) Expression of Sonic hedgehog and its putative role as a precursor cell mitogen in the developing mouse retina. Development 124:363-371.

Kolpak A, Zhang J, Bao ZZ (2005) Sonic hedgehog has a dual effect on the growth of retinal ganglion axons depending on its concentration. J Neurosci 25:3432-3441.

Locker M, Agathocleous M, Amato MA, Parain K, Harris WA, Perron M (2006) Hedgehog signaling and the retina: insights into the mechanisms controlling the proliferative properties of neural precursors. Genes Dev 20:3036-3048.

Lupo G, Liu Y, Qiu R, Chandraratna RA, Barsacchi G, He RQ, Harris WA (2005) Dorsoventral patterning of the Xenopus eye: a collaboration of Retinoid, Hedgehog and FGF receptor signaling. Development 132:17371748.

Manning L, Ohyama K, Saeger B, Hatano O, Wilson SA, Logan M, Placzek M (2006) Regional morphogenesis in the hypothalamus: a BMP-Tbx2 pathway coordinates fate and proliferation through Shh downregulation. Dev Cell 11:873-885.

Marcus RC, Shimamura K, Sretavan D, Lai E, Rubenstein JL, Mason CA (1999) Domains of regulatory gene expression and the developing optic chiasm: correspondence with retinal axon paths and candidate signaling cells. J Comp Neurol 403:346-358.

Marcus RC, Matthews GA, Gale NW, Yancopoulos GD, Mason CA (2000) Axon guidance in the mouse optic chiasm: retinal neurite inhibition by ephrin "A"-expressing hypothalamic cells in vitro. Dev Biol 221:132-147.
Nakagawa S, Brennan C, Johnson KG, Shewan D, Harris WA, Holt CE (2000) Ephrin-B regulates the ipsilateral routing of retinal axons at the optic chiasm. Neuron 25:599-610.

Okada A, Charron F, Morin S, Shin DS, Wong K, Fabre PJ, Tessier-Lavigne M, McConnell SK (2006) Boc is a receptor for sonic hedgehog in the guidance of commissural axons. Nature 444:369-373.

Petros TJ, Rebsam A, Mason CA (2008) Retinal axon growth at the optic chiasm: to cross or not to cross. Annu Rev Neurosci 31:295-315.

Petros TJ, Shrestha BR, Mason C (2009) Specificity and sufficiency of EphB1 in driving the ipsilateral retinal projection. J Neurosci 29:3463-3474.

Plump AS, Erskine L, Sabatier C, Brose K, Epstein CJ, Goodman CS, Mason CA, Tessier-Lavigne M (2002) Slit1 and Slit2 cooperate to prevent premature midline crossing of retinal axons in the mouse visual system. Neuron 33:219-232.

Sánchez-Camacho C, Bovolenta P (2008) Autonomous and non-autonomous Shh signalling mediate the in vivo growth and guidance of mouse retinal ganglion cell axons. Development 135:3531-3541.

Torres M, Gómez-Pardo E, Gruss P (1996) Pax2 contributes to inner ear patterning and optic nerve trajectory. Development 122:3381-3391.

Trousse F, Martí E, Gruss P, Torres M, Bovolenta P (2001) Control of retinal ganglion cell axon growth: a new role for Sonic hedgehog. Development 128:3927-3936.

Wallace VA, Raff MC (1999) A role for Sonic hedgehog in axon-to-astrocyte signalling in the rodent optic nerve. Development 126:2901-2909.

Wang Y, Dakubo GD, Thurig S, Mazerolle CJ, Wallace VA (2005) Retinal ganglion cell-derived sonic hedgehog locally controls proliferation and the timing of RGC development in the embryonic mouse retina. Development 132:5103-5113.

Williams SE, Mann F, Erskine L, Sakurai T, Wei S, Rossi DJ, Gale NW, Holt CE, Mason CA, Henkemeyer M (2003) Ephrin-B2 and EphB1 mediate retinal axon divergence at the optic chiasm. Neuron 39:919-935.

Williams SE, Grumet M, Colman DR, Henkemeyer M, Mason CA, Sakurai T (2006) A role for Nr-CAM in the patterning of binocular visual pathways. Neuron 50:535-547.

Yam PT, Langlois SD, Morin S, Charron F (2009) Sonic hedgehog guides axons through a noncanonical, Src-family-kinase-dependent signaling pathway. Neuron 62:349-362.

Zhang XM, Yang XJ (2001) Regulation of retinal ganglion cell production by Sonic hedgehog. Development 128:943-957. 